Article

\title{
Symmetry Breaking and Emergence of Directional Flows in Minimal Actomyosin Cortices
}

\author{
Sven K. Vogel ${ }^{1}$, Christian Wölfer ${ }^{2}$, Diego A. Ramirez-Diaz ${ }^{1,3}$, Robert J. Flassig ${ }^{2,4}$, \\ Kai Sundmacher ${ }^{2,5}$ and Petra Schwille ${ }^{1, * \mathbb{D}}$ \\ 1 Max Planck Institute of Biochemistry, Am Klopferspitz 18, D-82152 Martinsried, Germany; \\ svogel@biochem.mpg.de (S.K.V.); daramirez@g.harvard.edu (D.A.R.-D.) \\ 2 Max Planck Institute for Dynamics of Complex Technical Systems, Sandtorstr. 1, \\ D-39106 Magdeburg, Germany; woelfer@mpi-magdeburg.mpg.de (C.W.); \\ flassig@th-brandenburg.de (R.J.F.); sundmacher@mpi-magdeburg.mpg.de (K.S.) \\ 3 Graduate School of Quantitative Biosciences, Ludwig-Maximilians-Universität, Feodor-Lynen-Str. 25, \\ D-81377 Munich, Germany \\ 4 Department of Engineering, Brandenburg University of Applied Sciences, Magdeburger Str. 50, \\ D-14770 Brandenburg, Germany \\ 5 Institute for Process Engineering, Otto von Guericke University Magdeburg, Universitätsplatz 2, \\ D-39106 Magdeburg, Germany \\ * Correspondence: schwille@biochem.mpg.de
}

Received: 31 March 2020; Accepted: 31 May 2020; Published: 9 June 2020

\begin{abstract}
Cortical actomyosin flows, among other mechanisms, scale up spontaneous symmetry breaking and thus play pivotal roles in cell differentiation, division, and motility. According to many model systems, myosin motor-induced local contractions of initially isotropic actomyosin cortices are nucleation points for generating cortical flows. However, the positive feedback mechanisms by which spontaneous contractions can be amplified towards large-scale directed flows remain mostly speculative. To investigate such a process on spherical surfaces, we reconstituted and confined initially isotropic minimal actomyosin cortices to the interfaces of emulsion droplets. The presence of ATP leads to myosin-induced local contractions that self-organize and amplify into directed large-scale actomyosin flows. By combining our experiments with theory, we found that the feedback mechanism leading to a coordinated directional motion of actomyosin clusters can be described as asymmetric cluster vibrations, caused by intrinsic non-isotropic ATP consumption with spatial confinement. We identified fingerprints of vibrational states as the basis of directed motions by tracking individual actomyosin clusters. These vibrations may represent a generic key driver of directed actomyosin flows under spatial confinement in vitro and in living systems.
\end{abstract}

Keywords: bottom-up synthetic biology; motor proteins; pattern formation; self-organization

\section{Introduction}

In animal cells, cortical actomyosin motions including actomyosin flows have been proposed to drive cell locomotion, cytokinesis, left-right symmetry breaking during embryonic development of multicellular organisms, and cellular or tissue chirality [1-7]. Despite the omnipresent functions and implications of cortical actomyosin motions and flows, the exact molecular origins and fundamental determinants of these phenomena, in particular how local symmetry breaking within apparently isotropic actin cortices scales up into these flows, are far from being understood. In many eukaryotic model systems, myosin motors acting on actin filaments are proposed to play a key role in driving actomyosin dynamics in cytokinetic rings and cortical actomyosin flows [1,5,8-10]. Distinct manipulation of myosin and actin independent of other cellular processes is challenging as 
they both are functionally highly integrated cellular proteins. To elucidate whether directional flows may result spontaneously from active processes without being guided by the structural anisotropy of the cellular architecture, it is mandatory to explore these phenomena in well-controlled reconstituted systems [11,12]. Recent studies used an approach of encapsulating frog egg cell extracts in droplets, so manipulation of the protein players may be easier when compared with living systems $[13,14]$. Nevertheless, due to the compositional complexity of cell extracts is comparable to living model systems, it may be difficult to pinpoint the minimal set of necessary proteins to produce a certain phenomenon. High density regimes of nematic (filament alignment in parallel lines) cytoskeletal in vitro systems showed pattern formation and dynamics in a collective manner based on motor driven sliding mechanisms between long cytoskeletal filaments $[15,16]$. In contrast, actomyosin cortices and rings in living systems consist of non-polar and disordered actin filament networks and are coupled to the cell membrane $[17,18]$. By a combination of bottom-up in vitro experiments and theory, we identified a generic mechanism of how large-scale directional flow-like actomyosin motions spanning several hundred micrometers are generated by myosin motors in a disordered, membrane coupled, and isotropic cytoskeletal system in a confined spherical environment.

\section{Results}

To investigate the emergence of coordinated large-scale flows from spontaneous contractions in quasi-isotropic cortices, we made use of recently developed minimal actin cortices (MACs) $[19,20]$ and confined them in water-in-oil droplets using microfluidic emulsification on Polydimethylsiloxan (PDMS) chips. The chip design is shown in Figure 1A. (Note that we also performed emulsification simply by manually mixing the components, in order to rule out any suspicion of flows caused by the pneumatic microfluidic setup.) 1,2-di-(9Z-octadecenoyl)-sn-glycero-3-phosphocholine (DOPC) and biotinylated lipids were dissolved in mineral oil, and for droplet formation we used a pneumatic microfluidic system where the flow rate of each channel is individually controlled (Movie S1). The aqueous solution contained dissolved actin monomers, neutravidin, and myosin in a salt buffer system. To test the proper formation of a lipid monolayer that includes biotinylated lipids (DSPE-PEG-2000-Biotin), we started with the encapsulation of fluorescently labeled neutravidin (Oregon Green 488 Neutravidin) (Figure 1B-D). By analyzing the fluorescence intensity distribution throughout the droplets, we showed that neutravidin only binds to the lipid monolayer when the lipid oil mixture also contains biotinylated lipids (Figure 1C,D). This indicates that neutravidin does not bind non-specifically to the water/oil interface. Co-encapsulation of myosin motors with the actin monomer and anchor system in the presence of ATP resulted in the formation of a MAC. Inside the droplets, actin monomers started to polymerize and bind to the lipid monolayer that contained biotinylated lipids, thus forming an initially isotropic, i.e., spatially symmetric actomyosin cortex (Figure 1B,E, Movie S2). Concurrently, ATP-induced contraction of the myosin motors resulted in the symmetry breakage of the isotropic actin carpet and in the formation of actomyosin clusters (Figure 2A-C, Movie S3). Strikingly, within minutes, a large-scale directional movement emerged from these spherically confined actomyosin clusters, which we refer to as cortical actomyosin motion (CAM) (Figures 1E and 2B; Movies S3 and S5). Please note that in the absence of ATP or in the presence of a motor inhibitor no CAMs occurred (data not shown). The CAMs could last more than one hour and cover distances of several hundred micrometers before eventually ceasing, reaching a state with only minimal movements (Movies S3 and S5). Interestingly, these flow-like motions somewhat resemble cortical actomyosin flow-like motions in vivo, e.g., in the C. elegans embryo. We characterized the velocity of the actomyosin cluster movements by using particle image velocimetry [21] (Figure 2C; Movie S4). In order to test parameters that change the velocity of CAMs, we co-encapsulated a crowding agent (methylcellulose) at various concentrations and found a respective increase of the velocities in the presence of methylcellulose (Figure 2D, compare Movies S3 and S5). By increasing the effective concentration of actin and myosin at the lipid monolayer membrane interface, the overall cluster velocity was increased more than five times. 

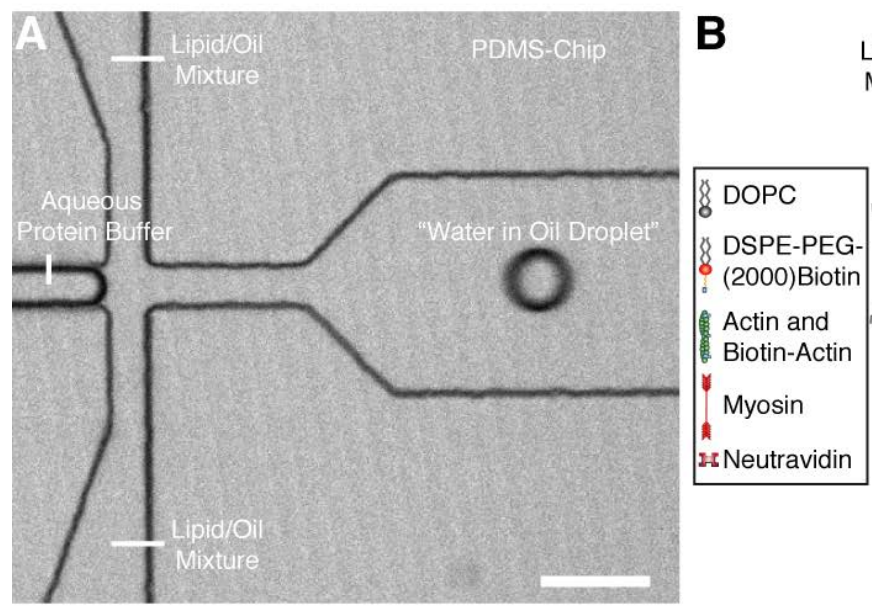
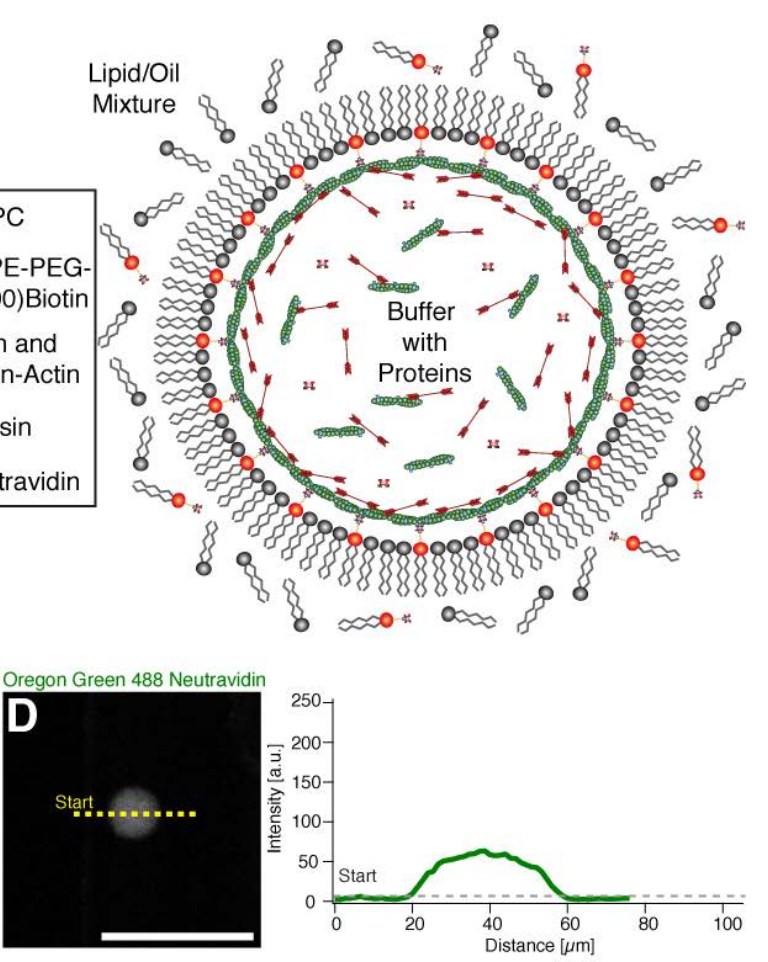

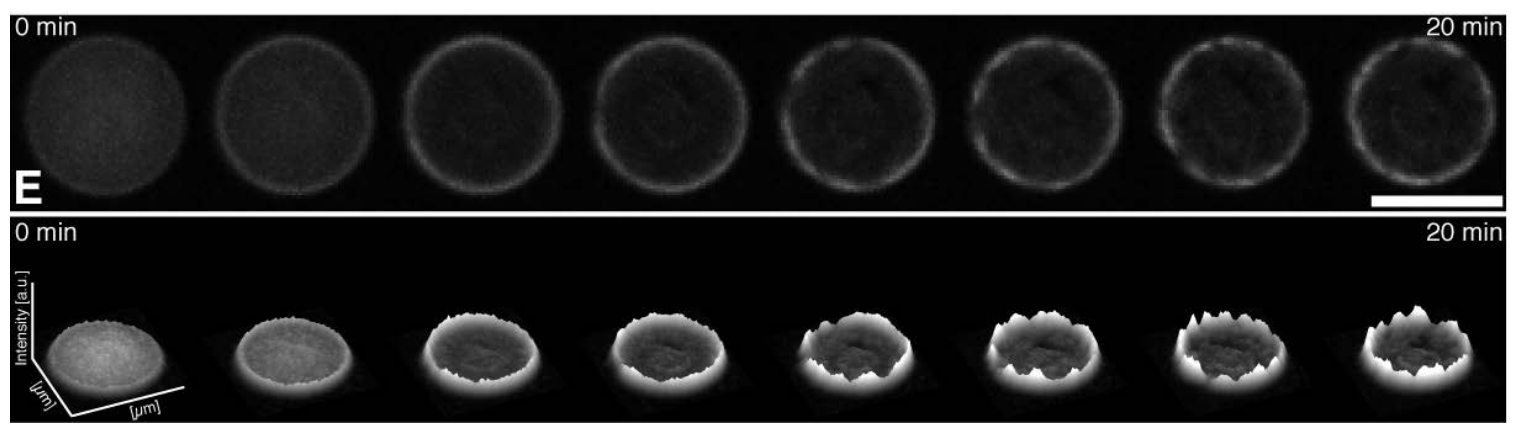

Figure 1. Encapsulation and actin cortex formation inside water in oil droplets. (A) Confocal image of a PDMS chip where the encapsulation of the buffer-protein system (see (B)) and formation of the water in oil droplet is shown (Movie S1). (B) Illustration depicting the formation of an actomyosin cortex. Neutravidin with its four binding sites may bind several actin filaments and biotinylated lipids. (C) Confocal image of the equatorial plane of a droplet with a lipid monolayer containing biotinylated lipids showing that encapsulated Oregon green labeled neutravidin binds to the lipid monolayer. Line profile of the fluorescence signal of the Oregon green labeled neutravidin shows two peaks which indicate binding of the neutravidin to the lipid monolayer interface of the droplet (right). (D) In contrast, Oregon green labeled neutravidin does not bind to the lipid monolayer and is distributed throughout the lumen of the droplet in the absence of biotinylated lipids. The fluorescence line profile shows no peaks in the absence of biotinylated lipids indicating the absence of unspecific binding to the lipid monolayer (right). (E) Confocal time-lapse images of encapsulated Alexa-488 labeled actin and myosin motors in the presence of ATP at the droplet equator plane. The formation of an actin cortex and actomyosin clusters is shown (upper row) (Movie S2). The respective fluorescent intensity profile indicates the formation of actomyosin clusters and shows their dynamics (lower row). Scale bars, $100 \mu \mathrm{m}$. 

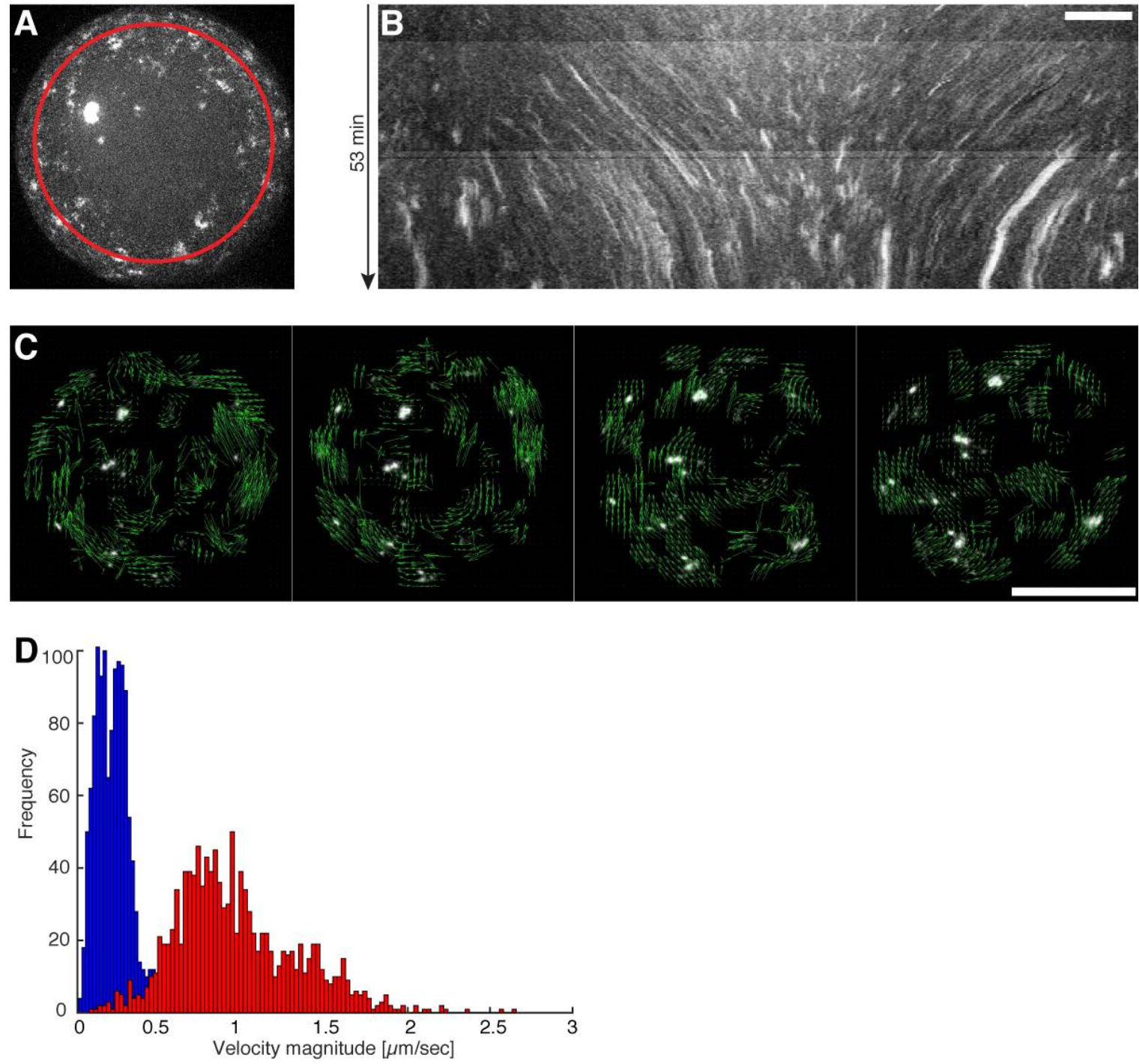

Figure 2. Directed movement of actomyosin clusters upon ATP dependent actomyosin contractions. (A) Maximum intensity projection from a half droplet confocal z-stack where Alexa-488 labeled actin clusters are visible (Movie S3). The red circle indicates the path of the generated kymograph. (B) A kymograph of the maximum intensity projected half sphere is shown where directed movements of individual clusters represented by distinct lines are visible. Scale bar, $10 \mu \mathrm{m}$. (C) Confocal time-lapse image sequence used for particle image velocimetry (PIV) by (21). Vectors (green arrows) indicate the flow direction of the directed movement of the actomyosin clusters (Movie S4). Scale bar, $50 \mu \mathrm{m}$. (D) Velocity profiles measured by PIV of droplets with (red) and without a crowding agent (blue) are shown (Movies S3 and S5).

\section{Theoretical Model of the Cortical Actomyosin Motions}

We then aimed to elucidate the mechanism behind myosin driven CAMs and complemented our experiments with a theoretical model based on biophysical first principles, i.e., without assuming an explicit mechanism a priori. We modeled the MAC droplet as a continuous, isotropic fluid. The interaction between actin and myosin was modeled by a simplified myosin cross-bridge cycle [22-24] where the force generating conformational change $\left(r_{1}\right)$ of the myosin head $(M)$ occurs immediately after the binding of filamentous actin (A) (Figure 3A). The actomyosin complex dissociates after binding and ATP hydrolysis reloads the myosin head into the active state $\left(\mathrm{r}_{2}\right)$. Typically, considered intermediate species only have a very short lifetime [25] and are therefore neglected. As the MACs were formed by polymerization in the absence of actin-regulating proteins [26-28], a rudimentary 
polymerization cycle [25] was added to the model. F-actin depolymerizes $\left(\mathrm{r}_{3}\right)$ into monomeric G-actin with bound ADP $\left(G_{D}\right)$, followed by a spontaneous nucleotide exchange $\left(r_{4}\right)$. The resulting ATP bound G-actin $\left(\mathrm{G}_{\mathrm{T}}\right)$ binds stronger to $\mathrm{F}$-actin $\left(\mathrm{r}_{5}\right)$ than $\mathrm{G}_{\mathrm{D}}$-actin $\left(\mathrm{r}_{6}\right)$, and therefore $\mathrm{G}_{\mathrm{T}}$-actin polymerization is dominant [29]. Both reaction systems are necessary to reasonably describe the local ATP consumption by polymerization and contraction. F-actin, cross-linked by myosin II molecules, forms a mesh-like cortex, causing an active viscoelastic material behavior with a rheological property combination of the Maxwell and Kelvin-Voigt models [30] considered in the momentum equation [31] (Supplemental Equation (S14)). To model force generation according to the myosin cross-bridge sub-model, the active stress was developed from a stress term of our previous study [32] considering the observed medium ATP-dependency of myosin pulls in MACs [20]. The distributed reaction network is described by a system of partial differential equations (PDEs) assuming diffusion $\left(G_{D}, G_{T}\right.$, ATP) or combined convection and diffusion flux $\left(\mathrm{A}, \mathrm{A}_{\mathrm{M}}, \mathrm{M}\right)$ (Supplemental Equations (S1)-(S6)). We reduced our analysis to a one-dimensional ring topology, modeling a section of the spherical droplet to improve and simplify the interpretation of the simulation results. To adopt experimental conditions of spontaneous symmetry breaking, the PDE system was simulated with an initially slightly inhomogeneous myosin distribution and revealed merging of small clusters into a gradually bigger main cluster, similar to experimental observations (Figure 3B,C) for the used parameter space, which is largely based on literature values (Table S2). The final non-symmetric cluster starts to move directionally in the one-dimensional ring system like a propagating wave (Figure 3B,C). Hereinafter, two feedback characteristics are described which are presumably essential for this movement. First, a non-symmetric contraction profile is preserved by an unbalanced consumption of ATP at the leading and trailing edge of the propagating cluster. Because of the locomotion and the ongoing network depolymerization, the cluster traces non-convective $G_{D}$-actin like a comet tail (Figure $S 1$ ). In the $G_{D}$-actin tail, ATP is additionally consumed for the nucleotide exchange of monomeric actin $\left(r_{4}\right)$. Thus, less ATP is available for the force-generating myosin cross-bridge cycle at the trailing edge of the wave and hence, less contractile stress is generated compared to the leading edge (Figure 3D). This local asymmetry is maintained despite consideration of the high diffusivity of ATP in an aqueous medium (Table S2). A reduction of the F-actin depolymerization leads to the loss of the $G_{D}$-actin tail the termination of the cluster locomotion (Figure S7). Additionally, locally reduced ATP distributions induce a change of locomotion direction as soon as the cluster reaches a reduced region. Here, more ATP is available at the trailing edge than at the leading edge and the asymmetry is reversed (Figure S8). Second, examining the fine structure of the simulated moving cluster reveals a vibration. It is driven by periodic depletion of the local ATP concentration, leading to oscillating active and passive mechanical stresses inside the cluster (Figure S3A). The cluster vibration passes through the following repetitive phases (Figure 3F): 
A

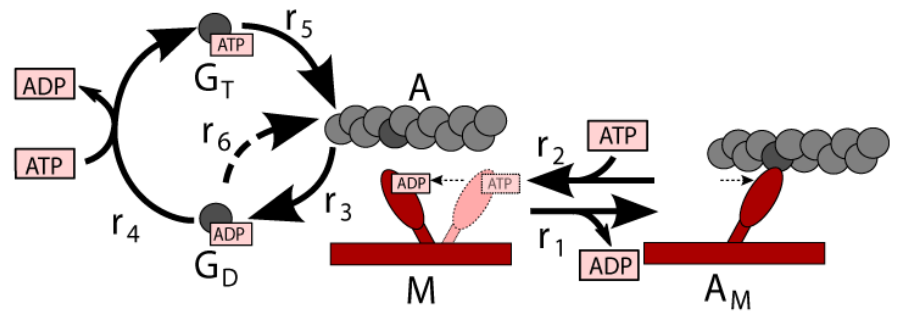

C

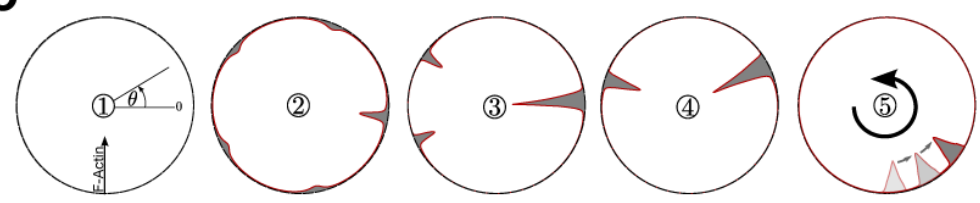

D

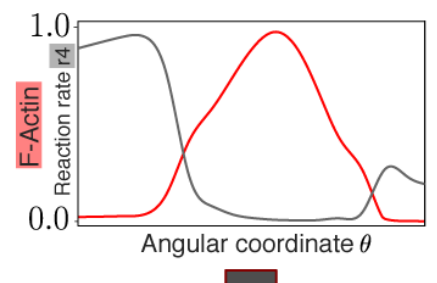

$+$
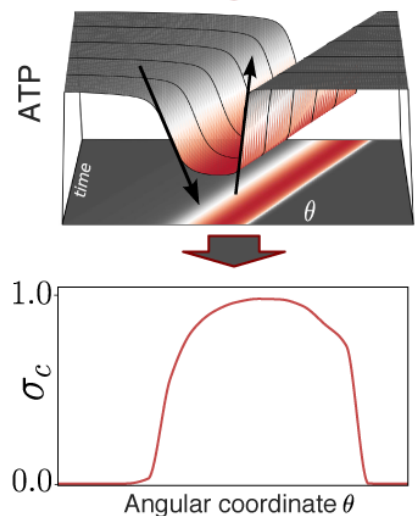

E

F Asymmetric contraction

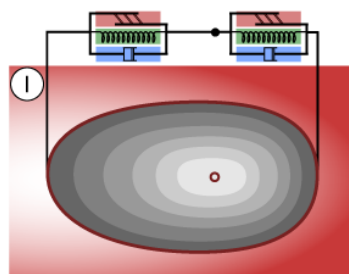

ATP Actin III Contractile stress
B

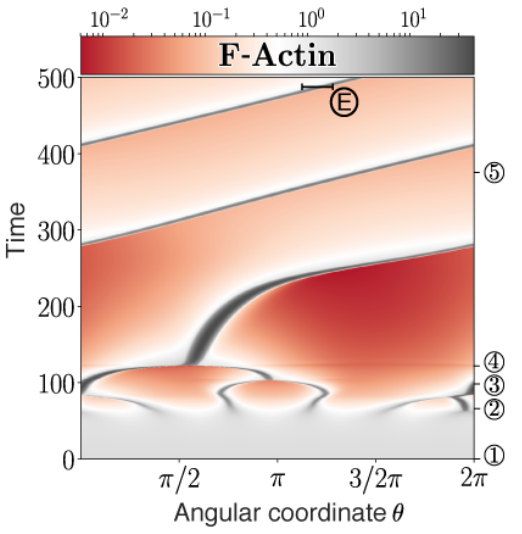

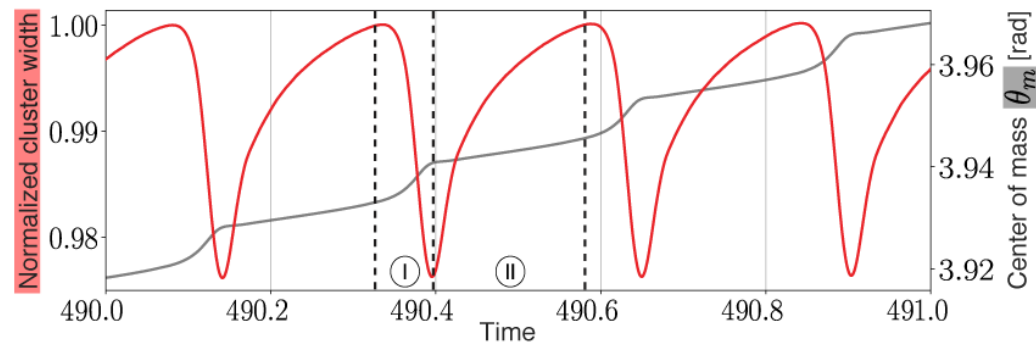

Beginning expansion

De novo contraction
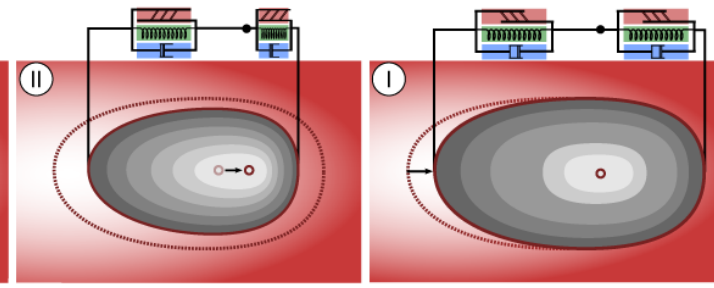

mmmmm Elastic stress $-\mathrm{F}$ Viscous stress

Figure 3. Modeling and simulation of actomyosin cluster motions reveal a propagation mechanism through active matter vibrations. (A) Kinetic reaction network with F-actin polymerization cycle and simplified myosin cross-bridge model. (B) Temporal development of a one-dimensional spatial F-actin distribution with color-coded local concentration. (C) Circular representation of distributed F-actin concentration according to the model topology for selected time points (marked in (B) with circles). (D) Non-symmetric contraction of a propagating cluster. Upper graph: Normalized distribution of reaction rate 4 (gray) compared to normalized F-actin cluster location (red). Middle graph: Non-symmetric ATP distribution around the cluster. Lower graph: Normalized asymmetric contractile stress pattern with color code representing ATP concentration according to the projection. (E) Vibration of an F-actin cluster. Evolution of normalized cluster width (red) and displacement of center of mass $\theta_{m}$ (gray) of F-actin in radiant. Cluster boundaries are defined as the points where the F-actin concentration exceeds the mean concentration. (F) Sketch of the cortical actin cluster migration mechanism with qualitative color-coded Actin and ATP gradients shows repetitive asymmetric contractions (I) and an expansion phase (II) resulting in a shift of the center of mass according to (D) indicated by a black arrow (middle panel) and displacement of the cluster indicated by an arrow (right panel).

I. Contraction Phase: In the beginning of the contraction phase, a high ATP concentration inside the cluster results in an increase of the contractile stress accompanied by an increase of elastic stress. With progressing cluster compaction, the actomyosin species gets locally accumulated, causing stronger contractile stress and increased local ATP consumption. 
II. Expansion Phase: Increasing local ATP depletion and network strain leads to a local dominance of elastic stress resulting in an expansion of the cluster. Because of the reduced network densities inside of the cluster and therefore reduced energy consumption, ATP flows in diffusively and increases the local ATP level, followed by a de novo contraction phase.

In addition, the asymmetric contraction pattern causes an accelerated displacement of the center of mass during the contraction phase (Figure 3E,F). Owing to viscoelastic material properties and asymmetric creep, the center of mass is not pushed back to the initial position during the expansion phase, a result of the asymmetric contraction pattern. To investigate the wave propagation mechanism, we assumed a constant diffusive ATP supply. Since the active stress is ATP-driven, the wave propagation ceases, owing to the global ATP depletion (Figure S2). Hence, cluster vibrations due to local ATP depletion and asymmetric cluster contractions are the main drivers of single cluster motions. Parameter studies show that these motions occur in a large parameter space, encompassing several orders of magnitude, and are therefore robust for the assumed parameters (Figure S9). In contrast to previous theoretical studies $[33,34]$, where the required asymmetry of a self-propagating cluster is caused by polar actin bundles, our suggested model is also able to explain cluster migrations of disordered actin filaments that can be expected in an isotropic cortex.

To find experimental evidence for the theoretically predicted vibrations of the actomyosin clusters, we automatically tracked their directional movement (Figure 4A). In addition, by analyzing the directed movement of the individual actomyosin clusters, we noticed that rotation of clusters around their centers of mass correlated with a change in direction displacement (Figure 4A,B; Movies S6 and S7). This can be explained with torque produced by an imbalance of forces perpendicular to the translational trajectory. On the other hand, the model predicts vibrations with a certain oscillation period (Figure 3E). Evidence for such vibrations in our experimental conditions can be found independently of the sampling rate. For example, a sinusoidal signal with a specific frequency can be measured at under-sampling conditions (Figure S5). The reconstructed signals still conserve the periodic behavior reflected by the Fourier analysis, yet not maintaining the original frequency (Figure S5). To find evidence for vibrations inside the clusters as predicted by our theory, we analyzed image sequences to measure fluctuations in the distance between the geometrical and the intensity-weighted center of mass of several clusters. The geometrical center of mass was computed by regular segmentation and binarization defining the geometry of the cluster. In contrast, the intensity-weighted center of mass was calculated over the same geometry but having the camera-intensity as the statistical weight of every pixel. Since clusters have various geometries, we defined a characteristic length for the clusters as the square root of the area. This quantity allowed us to measure changes between the geometrical and intensity centers of mass with respect to their characteristic length. As a result, we were able to combine fluctuations of myosin clusters with different sizes and experimental conditions (Figure S6). Fourier analysis of these combined fluctuations strikingly indicates the existence of vibrational states (Figure $4 \mathrm{D}$, red, blue and yellow curves) which are clearly distinguishable from static conditions (Figure 4D, grey curve) or acquisition and camera artifacts by measuring the background between moving clusters (data not shown). We interpret these vibrational states as fingerprints of the theoretically predicted vibrations that provide a mechanism for the directed and rotational large-scale movements of the actomyosin clusters in silico and in vitro. Under crowding conditions where CAMs show the highest velocities, we found higher amplitudes for the same observed frequencies in comparison to the other tested conditions (Figure 4D). This finding implies that larger amplitudes correlate with higher velocities as expected for vibrations-based movements. Since the crowder increases the effective concentration of actin and myosin at the lipid monolayer membrane interface, we conclude that higher actomyosin concentrations may lead to higher cluster velocities and therefore to larger amplitudes of the vibrations. 

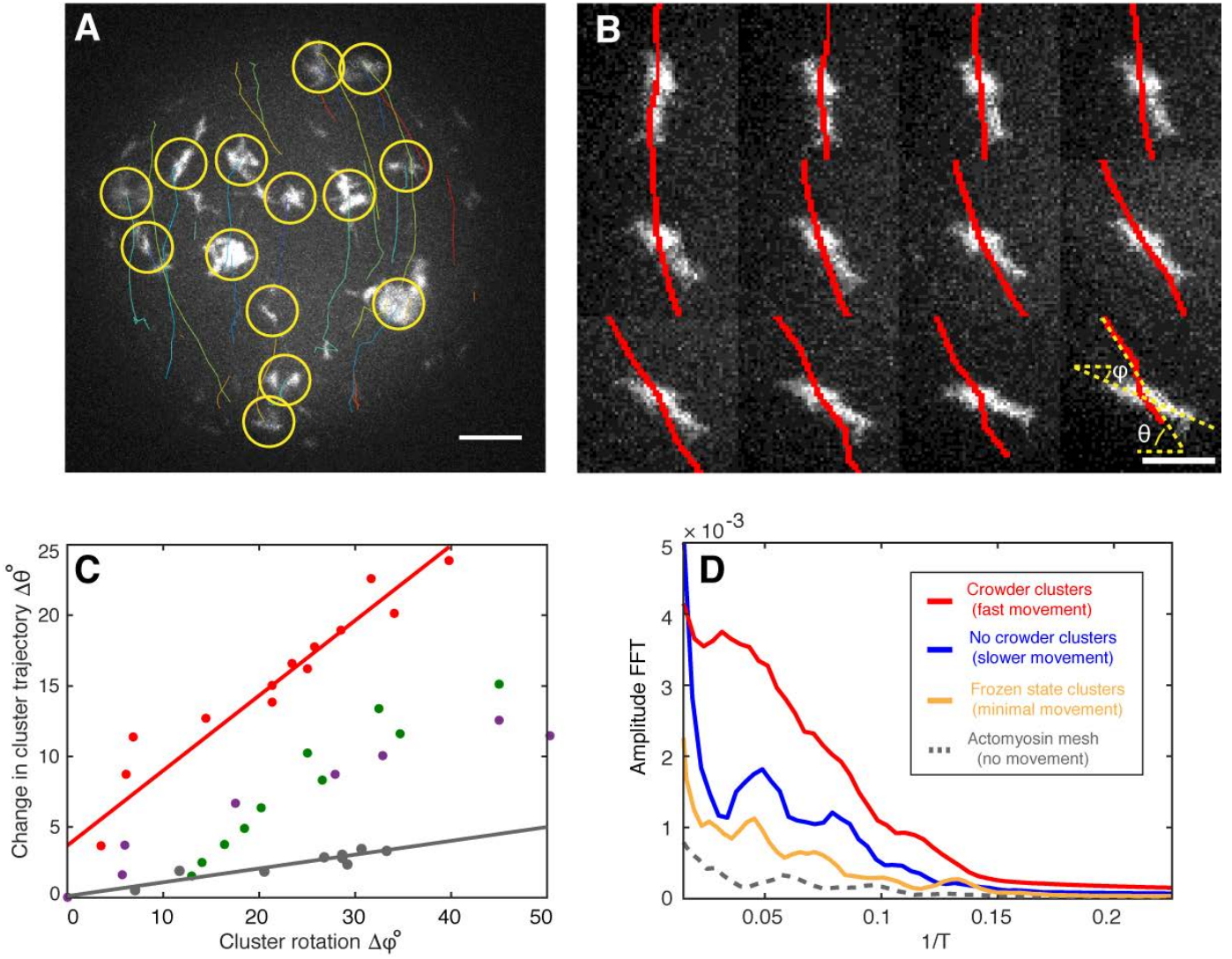

Figure 4. Fingerprints of actomyosin cluster vibrations during their directed movements. (A) A maximum intensity projection of confocal images of a half sphere during cortical actomyosin cluster movements is shown. Tracked actin clusters are marked by circles (yellow) and their trajectories shown (various colors, Movie S6). (B) Changes in the angle trajectory (red lines, $\theta$ ) agree with cluster rotations along its center of mass $(\phi)$ (left, Movie S7). Scale bar, $4 \mu \mathrm{m}$. (C) Analysis of four independent clusters (various colors) suggest that rotation influences cluster steering and then a change in trajectory $(\Delta \theta)$. The red data points correspond to the montage shown in (B). (D) The fast Fourier transformation (FFT) indicates the presence of vibrational states for moving actomyosin clusters (red, blue, and yellow) different from static systems and acquisition artifacts (grey). The higher amplitude for the crowder condition with the highest cluster velocities of all measured systems implies that larger amplitudes correlate with higher velocities. Scale bar, $10 \mu \mathrm{m}$.

\section{Discussion}

Based on our suggested mechanism for the directional motion of an individual cluster, we consider the possibility of an alignment of motion of a group of clusters in one direction to result from potential cluster interactions via the cortical actin network, thereby explaining the observed flow-like group behavior of the actomyosin clusters. Alternatively, a macroscopically homogeneous quasi-symmetric actin carpet is initially formed, as shown in our experiments (see Figure 1E, Movies S2 and S3). Since the resulting clusters are initially connected via the isotropic actin mesh, the random asymmetry of the largest cluster would determine the direction of movement of the entire cluster group, in the absence of coordinating processes. Each cluster of the group is thus impressed with a direction of movement or an asymmetric gradient profile originating from the respective monomer tail. In conclusion, our theory suggests that the direct translational and rotational movements of actomyosin clusters originate from an imbalance of oscillatory contractile stresses within the individual actomyosin clusters. Fourier analysis of our experimental data indicates the existence of vibrational states that drive the directional movements of individual actomyosin clusters and the formation of flow-like CAMs in the spherical confinement of the active matter droplets. Hence, for future work it will be of great interest to investigate 
whether these vibrational states can be also identified in cellular model systems that exhibit myosin driven actomyosin motions, e.g., in cytokinetic actomyosin rings or in cortical actomyosin flows.

\section{Methods}

Additional Information and details of the model as well as all methods and experimental procedures and movie legends can be found in the Supporting Information Appendix.

Supplementary Materials: The following are available online at http://www.mdpi.com/2073-4409/9/6/1432/s1, Supporting Information (word document), Movie S1: Encapsulation and water in oil droplet formation, Movie S2: Actin cortex formation and myosin contraction, Movie S3: Directional actomyosin flows, Movie S4: Particle Image Velocimetry (PIV) of moving actomyosin clusters, Movie S5: Enhanced directional actomyosin flows through crowding, Movie S6: Tracking of individual actomyosin clusters for determining fingerprints of cluster vibrations, Movie S7: Rotation of actomyosin clusters.

Author Contributions: Conceptualization experiments: S.K.V. and P.S.; Conceptualization theory: C.W. and R.J.F. Methodology experiments: S.K.V., D.A.R.-D.; Methodology theory: C.W. and R.J.F.; Software experiments: S.K.V and D.A.R.-D.; Software theory: C.W. and R.F.F.; Validation: S.K.V., C.W., D.A.R.-D. and R.J.F.; Formal analysis experiments: S.K.V., D.A.R.-D.; Formal analysis theory: C.W. and R.J.F.; Investigation experiments: S.K.V.; Investigation theory: C.W.; Resources, S.K.V.; Writing-original draft preparation: S.K.V., C.W., R.J.F., D.A.R.-D.; writing-review and editing, P.S. and K.S.; Visualization: S.K.V. and C.W.; Funding acquisition: P.S. and K.S. All authors have read and agreed to the published version of the manuscript.

Funding: This research was funded by MaxSynBio, a joint research network sponsored by the German Federal Ministry of Education and Research (BMBF) and the MaxPlanck Society (MPG).

Acknowledgments: We thank Chase Broedersz for discussions and critical reading of the manuscript. We are grateful for the financial support by the MaxSynBio consortium jointly funded by the Federal Ministry of Education and Research of Germany and the Max Planck Society, the Daimler und Benz foundation (Project Grant PSBioc 8216), the Gottfried Wilhelm Leibniz-Program of the DFG (SCHW 716/8-1) and the support of the Graduate School of Quantitative Biosciences Munich.

Conflicts of Interest: The authors declare no conflict of interest.

\section{References}

1. Bray, D.; White, J.G. Cortical flow in animal cells. Science 1988, 239, 883-888. [CrossRef]

2. Sedzinski, J.; Biro, M.; Oswald, A.; Tinevez, J.Y.; Salbreux, G.; Paluch, E. Polar actomyosin contractility destabilizes the position of the cytokinetic furrow. Nature 2011, 476, 462-466. [CrossRef]

3. Khaliullin, R.N.; Green, R.A.; Shi, L.Z.; Gomez-Cavazos, J.S.; Berns, M.W.; Desai, A.; Oegema, K. A positive-feedback-based mechanism for constriction rate acceleration during cytokinesis in Caenorhabditis elegans. Elife 2018, 7, e36073. [CrossRef] [PubMed]

4. Naganathan, S.R.; Middelkoop, T.C.; Furthauer, S.; Grill, S.W. Actomyosin-driven left-right asymmetry: From molecular torques to chiral self organization. Curr. Opin. Cell Biol. 2016, 38, 24-30. [CrossRef] [PubMed]

5. Wollrab, V.; Thiagarajan, R.; Wald, A.; Kruse, K.; Riveline, D. Still and rotating myosin clusters determine cytokinetic ring constriction. Nat. Commun. 2016, 7, 11860. [CrossRef] [PubMed]

6. Callan-Jones, A.C.; Voituriez, R. Actin flows in cell migration: From locomotion and polarity to trajectories. Curr. Opin. Cell Biol. 2016, 38, 12-17. [CrossRef]

7. Klughammer, N.; Bischof, J.; Schnellbacher, N.D.; Callegari, A.; Lenart, P.; Schwarz, U.S. Cytoplasmic flows in starfish oocytes are fully determined by cortical contractions. PLoS Comput. Biol. 2018, 14, e1006588. [CrossRef] [PubMed]

8. Munro, E.; Nance, J.; Priess, J.R. Cortical flows powered by asymmetrical contraction transport PAR proteins to establish and maintain anterior-posterior polarity in the early C. elegans embryo. Dev. Cell 2004, 7, 413-424. [CrossRef]

9. Naganathan, S.R.; Furthauer, S.; Nishikawa, M.; Julicher, F.; Grill, S.W. Active torque generation by the actomyosin cell cortex drives left-right symmetry breaking. Elife 2014, 3, e04165. [CrossRef]

10. Nishikawa, M.; Naganathan, S.R.; Julicher, F.; Grill, S.W. Controlling contractile instabilities in the actomyosin cortex. Elife 2017, 6, e19595. [CrossRef]

11. Fletcher, D. Which biological systems should be engineered? Nature 2018, 563, 177-179. [CrossRef] 
12. Schwille, P. Bottom-up synthetic biology: Engineering in a tinkerer's world. Science 2011, 333, $1252-1254$. [CrossRef]

13. Suzuki, K.; Miyazaki, M.; Takagi, J.; Itabashi, T.; Ishiwata, S. Spatial confinement of active microtubule networks induces large-scale rotational cytoplasmic flow. Proc. Natl. Acad. Sci. USA 2017, 114, 2922-2927. [CrossRef] [PubMed]

14. Tan, T.H.; Malik-Garbi, M.; Abu-Shah, E.; Li, J.; Sharma, A.; MacKintosh, F.C.; Keren, K.; Schmidt, C.F.; Fakhri, N. Self-organized stress patterns drive state transitions in actin cortices. Sci. Adv. 2018, 4, eaar2847. [CrossRef] [PubMed]

15. Schaller, V.; Weber, C.; Semmrich, C.; Frey, E.; Bausch, A.R. Polar patterns of driven filaments. Nature 2010, 467, 73-77. [CrossRef] [PubMed]

16. Keber, F.C.; Loiseau, E.; Sanchez, T.; DeCamp, S.J.; Giomi, L.; Bowick, M.J.; Marchetti, M.C.; Dogic, Z.; Bausch, A.R. Topology and dynamics of active nematic vesicles. Science 2014, 345, 1135-1139. [CrossRef]

17. Svitkina, T.M.; Borisy, G.G. Correlative light and electron microscopy of the cytoskeleton of cultured cells. Methods Enzymol. 1998, 298, 570-592.

18. Stachowiak, M.R.; Laplante, C.; Chin, H.F.; Guirao, B.; Karatekin, E.; Pollard, T.D.; O'Shaughnessy, B. Mechanism of cytokinetic contractile ring constriction in fission yeast. Dev. Cell 2014, 29, 547-561. [CrossRef]

19. Vogel, S.K.; Heinemann, F.; Chwastek, G.; Schwille, P. The design of MACs (minimal actin cortices). Cytoskeleton 2013, 70, 706-717. [CrossRef]

20. Vogel, S.K.; Petrasek, Z.; Heinemann, F.; Schwille, P. Myosin Motors Fragment and Compact Membrane-Bound Actin Filaments. eLife 2013, 2013, e00116. [CrossRef]

21. Thielicke, W. PIVlab-Towards User-friendly, Affordable and Accurate Digital Particle Image Velocimetry in MATLAB. J. Open Res. Softw. 2014, 2, e30. [CrossRef]

22. Huxley, H.E. The mechanism of muscular contraction. Science 1969, 164, 1356-1365. [CrossRef] [PubMed]

23. Lymn, R.W.; Taylor, E.W. Mechanism of adenosine triphosphate hydrolysis by actomyosin. Biochemistry 1971, 10, 4617-4624. [CrossRef] [PubMed]

24. Spudich, J.A. The myosin swinging cross-bridge model. Nat. Rev. Mol. Cell Biol. 2001, 2, 387-392. [CrossRef] [PubMed]

25. Howard, J. (Ed.) Mechanics of Motor Proteins and the Cytoskeleton; Sinauer Associates Inc.: Sunderland, MA, USA, 2001.

26. Pollard, T.D.; Borisy, G.G. Cellular motility driven by assembly and disassembly of actin filaments. Cell 2003, 112, 453-465. [CrossRef]

27. Pollard, T.D.; Cooper, J.A. Actin, a central player in cell shape and movement. Science 2009, 326, $1208-1212$. [CrossRef]

28. Blanchoin, L.; Boujemaa-Paterski, R.; Sykes, C.; Plastino, J. Actin dynamics, architecture, and mechanics in cell motility. Physiol. Rev. 2014, 94, 235-263. [CrossRef]

29. Pollard, T.D.; Goldberg, I.; Schwarz, W.H. Nucleotide exchange, structure, and mechanical properties of filaments assembled from ATP-actin and ADP-actin. J. Biol. Chem. 1992, 267, 20339-20345.

30. Mogilner, A. Mathematics of cell motility: Have we got its number? J. Math. Biol. 2009, 58, 105-134. [CrossRef]

31. Lewis, O.L.; Guy, R.D.; Allard, J.F. Actin-myosin spatial patterns from a simplified isotropic viscoelastic model. Biophys. J. 2014, 107, 863-870. [CrossRef]

32. Wolfer, C.; Vogel, S.K.; Mangold, M. A curvilinear Model Approach: Actin Cortex Clustering Due to ATP-induced Myosin Pulls. IFAC Pap. 2016, 49, 103-108. [CrossRef]

33. Kruse, K.; Camalet, S.; Julicher, F. Self-propagating patterns in active filament bundles. Phys. Rev. Lett. 2001, 87, 138101. [CrossRef] [PubMed]

34. Kreten, F.H.; Hoffmann, C.; Riveline, D.; Kruse, K. Active bundles of polar and bipolar filaments. Phys. Rev. E 2018, 98, 012413. [CrossRef] [PubMed]

(C) 2020 by the authors. Licensee MDPI, Basel, Switzerland. This article is an open access article distributed under the terms and conditions of the Creative Commons Attribution (CC BY) license (http://creativecommons.org/licenses/by/4.0/). 